\title{
Neuronal activity in vivo enhances functional myelin repair
}

\author{
Fernando C. Ortiz, ${ }^{1,2,3}$ Chloé Habermacher, ${ }^{1,2,4}$ Mariana Graciarena, ${ }^{5}$ Pierre-Yves Houry, ${ }^{1,2}$ \\ Akiko Nishiyama, ${ }^{6}$ Brahim Nait Oumesmar, ${ }^{5}$ and María Cecilia Angulo $0^{1,2,4}$ \\ ${ }^{1}$ INSERM U1128, Paris, France. ${ }^{2}$ Université Paris Descartes, Sorbonne Paris Cité, Paris, France. ${ }^{3}$ Instituto de Ciencias \\ Biomédicas, Facultad de Ciencias de la Salud, Universidad Autónoma de Chile, Santiago, Chile. ${ }^{4}$ Institute of Psychiatry and \\ Neuroscience of Paris, INSERM U1266, Paris, France. Institut du Cerveau et de la Moelle épinière, Sorbonne Université, \\ INSERM U1127, CNRS UMR 7225, Paris, France. 'Department of Physiology and Neurobiology, University of Connecticut, \\ Storrs, Connecticut, USA.
}

In demyelinating diseases, such as multiple sclerosis, demyelination of neuronal fibers impairs impulse conduction and causes axon degeneration. Although neuronal activity stimulates oligodendrocyte production and myelination in normal conditions, it remains unclear whether the activity of demyelinated axons restores their loss of function in a harmful environment. To investigate this question, we established a model to induce a moderate optogenetic stimulation of demyelinated axons in the corpus callosum at the level of the motor cortex in which cortical circuit activation and locomotor effects were reduced in adult freely moving mice. We demonstrate that a moderate activation of demyelinated axons enhances the differentiation of oligodendrocyte precursor cells onto mature oligodendrocytes but only under a repeated stimulation paradigm. This activity-dependent increase in the oligodendrocyte pool promotes an extensive remyelination and functional restoration of conduction, as revealed by ultrastructural analyses and compound action potential recordings. Our findings reveal the need for preserving an appropriate neuronal activity in the damaged tissue to promote oligodendrocyte differentiation and remyelination, likely by enhancing axon-oligodendroglia interactions. Our results provide new perspectives for translational research using neuromodulation in demyelinating diseases.

Authorship note: $\mathrm{FCO}$ and $\mathrm{CH}$ contributed equally to this work.

Conflict of interest: The authors have declared that no conflict of interest exists.

Copyright: (c) 2019 American Society for Clinical Investigation

Submitted: July 9, 2018 Accepted: March 19, 2019 Published: May 2, 2019.

Reference information: /CI Insight. 2019;4(9):e123434. https://doi. org/10.1172/ji.insight.123434.

\section{Introduction}

In multiple sclerosis (MS), impaired impulse conduction arises when immune-mediated demyelination destroys the myelin sheaths and causes the death of myelin-forming oligodendrocytes (OLs) and progressive axon loss (1). However, production of newly formed OLs and some extent of myelin repair can occur in this demyelinating disease. Unfortunately, remyelination is often incomplete and ultimately fails in inducing functional recovery of patients with long-standing and progressive disease (1). Oligodendrocyte precursor cells (OPCs) are the main source of remyelinating OLs in demyelinated lesions. In some MS lesions populated by OPCs, remyelination fails, probably because the cell microenvironment is highly perturbed and axons are poorly receptive for remyelination $(2,3)$. Therefore, the mere presence of OPCs in a lesion is insufficient to stimulate a regenerative process. Importantly, neuronal activity is critical for the regulation of OPC proliferation, differentiation, and myelination in the normal central nervous system (CNS) (4-10). Although the effect of neuronal activity on remyelination has been less studied, neuronal activity could also be a key external factor in enhancing myelin regeneration. A role of neuronal activity in endogenous remyelination is suggested by the effect of in vivo perfusion of the $\mathrm{Na}^{+}$channel blocker tetrodotoxin (TTX), which increases the density of proliferating OPCs and reduces remyelination in a context of acute, toxin-induced demyelinated lesions (11). However, whether and which pattern of activity of demyelinated axons in an adverse cellular environment could have a beneficial effect during myelin regeneration is unknown.

If neuronal activity is critical to support remyelination, increasing the activity of demyelinated axons should improve this process. In cell cultures, low-frequency electrical stimulation of dorsal root ganglion (DRG) axons is associated with reduced number of myelin segments (12), while increasing the stimulation to physiological firing rates for DRG neurons reduces OPC proliferation, favoring their differentiation onto myelinating OLs (13). Under normal in vivo conditions, however, distinct and sometimes contradictory 
effects of neuronal activity have been described. The most accepted notion is that neuronal stimulation in freely moving mice induces a rise in OPC proliferation that is followed by their differentiation into newly generated OLs $(6,7,9,14)$. However, different intensities, frequencies, and durations of neuronal activity in distinct in vivo preparations can also (a) trigger OPC differentiation without a prior proliferative process (14), (b) shift the balance toward more OPC proliferation (6), or (c) lead to no changes in the number of cycling OPCs (9). Considering these variable results in normal conditions, it is thus difficult to predict which activity pattern of demyelinated fibers could promote OL differentiation and myelin repair in pathological conditions.

To investigate whether neuronal activity can enhance oligodendrocyte differentiation and restore myelin regeneration in demyelinated lesions, we established a model to stimulate demyelinated fibers using an optogenetic-based approach in freely moving mice after a lysophosphatidylcholine-induced (LPC-induced) focal demyelination of corpus callosum at the level of the motor cortex. We adjusted the stimulation parameters to obtain a restricted neuronal activation that does not cause changes in motor behavior but triggers action potentials in demyelinated axons. We studied the impact of neuronal activity in modulating different stages of remyelination by analyzing OPC proliferation and differentiation, the extent of myelin regeneration, and the functional recovery of the fibers. We found that, after an acute demyelination, neuronal activity in vivo enhances the differentiation of OPCs onto myelinating OLs, inducing a remarkable ultrastructural and functional improvement but only under a repeated stimulation paradigm. Our results highlight the importance of preserving a controlled, moderate, and recurrent activity of demyelinating axons to improve myelin regeneration.

\section{Results}

Model for moderate optogenetic stimulation of demyelinated callosal fibers in vivo. In Thy1-ChR2-YFP mice, layer $\mathrm{V}$ pyramidal neurons of the neocortex selectively express the light-sensitive channel channelrhodopsin-2 (ChR2) fused to yellow fluorescent protein (YFP) under the Thy1 promoter (15). In the motor cortex, these neurons are highly myelinated, thus conveying rapid information outside this region (16) and directly contributing to the execution of voluntary movements. In line with this, their optogenetic stimulation with a fiber optic placed at the pial surface in freely moving mice triggers a locomotor behavior during blue light stimulation trains characterized by circular, stereotyped displacements and sometimes followed by epileptic-like seizures (ref. 6 and Supplemental Figure 1; supplemental material available online with this article; https://doi.org/10.1172/jci.insight.123434DS1). This stimulation provides a broad activation of neuronal circuits involved in locomotor behavior. Changes in motor activity constitute a convenient readout to assess the efficiency of photostimulation in normal conditions (Supplemental Figure 1). However, this wide neuronal network activation seems less suitable to study local repair mechanisms because its off-target effects alter both global circuit functioning and animal behavior.

Here we performed LPC-induced demyelinated lesions in corpus callosum of adult Thy1-ChR2-YFP mice at the level of the motor cortex. To avoid a wide activation of motor circuits and ensure the targeting of demyelinated axons inside LPC-induced lesions, we implanted a mini-optic fiber that extended to deep cortical layers and whose tip lay above LPC-induced lesions (Figure 1A). Contrary to the stimulation at the pial surface (Supplemental Figure 1), we found that unilateral 30-second light pulse trains delivered at $20 \mathrm{~Hz}$ every 4.5 minutes did not induce modifications in locomotor behavior when comparing stimulated and unstimulated (control) mice over 50 minutes (10 trains; Figure 1, B and C). Furthermore, no significant changes were observed when analyzing 30-second periods before, during, and after each stimulation train (Figure 1D; $P>0.05$ for turning tendency, traveled distance, and mean speed, respectively, analyzed for 10 consecutive trains, 2-way ANOVA followed by Bonferroni's multiple-comparison test; $n=5$ mice). In the absence of behavioral changes, we verified that the stimulation was efficient by implanting an optrode accommodating a recording electrode inside the demyelinated lesion. Light-evoked local field potentials (LFPs) were recorded for each light pulse of $10 \mathrm{~ms}$ in long trains up to 30 seconds in anesthetized Thy1ChR2-YFP mice but were absent in wild-type mice (Figure 1E; Supplemental Figure 2, A and B). We also confirmed that layer $\mathrm{V}$ pyramidal neurons in acute cortical slices triggered action potentials with high fidelity during 30-second stimulation trains at $20 \mathrm{~Hz}$, a frequency compatible with their average firing rate in the motor cortex (17) (Supplemental Figure 2, C and D).

After in vivo light stimulation, LPC-induced lesions were recognized in brain tissue sections by the lack of myelin basic protein (MBP) upon immunostaining (Figure 1F). Only lesions showing demyelinated 
$\mathrm{YFP}^{+}$axons were kept for analysis to avoid those with potential mechanical damage induced during LPC injection (Figure 1F). Although the size of the lesions and number of cells expressing the oligodendroglia cell lineage marker Olig2 was very variable (Supplemental Figure 3, A-C), the Olig2 ${ }^{+}$cell density was constant among lesions, allowing for comparative analysis between conditions (Supplemental Figure 3D). Altogether, these results validate our in vivo model for moderate optogenetic stimulation of demyelinated, ChR2-expressing axons in LPC-induced lesions without causing major behavioral locomotor changes.

A photostimulation session leads to a rapid increase in the OPC pool inside demyelinated lesions. Because the LPC-induced demyelination model elicits a synchronized and successive response of OPCs and OLs (18, 19), the effect of increased neuronal activity can be evaluated at different stages of the demyelination/remyelination processes. We first assessed the effect of an increased demyelinated axon activity on OPC density after a single photostimulation session at 7 days after injection (dpi), a time point that corresponds to the tail end of OPC proliferation and the beginning of OPC differentiation that is expected to occur spontaneously in the absence of photostimulation $(18,19)$. Mice were photostimulated for 3 hours with 10-ms light pulses applied at $20 \mathrm{~Hz}$ during 30 seconds every 4.5 minutes and perfused 1 hour after the end of photostimulation (Figure 2A). A triple immunostaining was performed to simultaneously identify the lack of myelin in lesions of corpus callosum (MBP), all OL lineage cells (Olig2), and mature OLs (CC1). We considered Olig2 ${ }^{+} \mathrm{CC}^{-}$cells as OPCs and Olig2 ${ }^{+} \mathrm{CC}^{+}$cells as differentiated OLs (Figure $2 \mathrm{~B}$ ). We found a 1.61-fold increase of OPC density in photostimulated lesions compared with control lesions but observed no differences in OL density (Figure 2C). Consistent with previous findings in normal conditions (6), the increased OPC density in the ipsilateral side was not accompanied by changes in OPC or OL populations in the contralateral side (Supplemental Figure 4A). In addition, we observed that only $1.281 \pm 0.77 \%$ of Olig2 ${ }^{+}$cells coexpress the astrocytic marker glial fibrillary acidic protein (GFAP) in photostimulated lesions ( $n=5$, not shown), confirming that the observed cell density increase was restricted to the OPC population. Our results show that a transient increase in the activity of demyelinated axons is sufficient to rapidly raise the OPC density inside demyelinated lesions.

Several reports have shown that, during normal myelination, neuronal activity increases the number of cycling OPCs, as revealed by EdU staining that labels proliferative cells in the $\mathrm{S}$ phase of the cell cycle $(6,9,14)$. By performing 2 injections of EdU at the beginning and at the end of the photostimulation session (Figure 2A), we explored whether a similar cellular mechanism could explain the rapid, activity-dependent increase of OPC density in demyelinated lesions at $7 \mathrm{dpi}$. First, we found that most of the EdU ${ }^{+}$cells were Olig2- in control and photostimulated lesions and did not display any density change upon photostimulation (Figure 2, D and E). Although no changes were observed for these proliferative cells that should mainly comprise astrocytes and microglia, neuronal activity could affect each of these populations independently. We therefore performed specific experiments to follow proliferative microglia and astrocytes inside lesions (Supplemental Figure 5). No differences were observed in the $\mathrm{EdU}^{+} \mathrm{IBA}^{+}$micrgolia or $\mathrm{EdU}^{+} \mathrm{GFAP}^{+}$astrocyte densities between control and photostimulated mice, indicating that neuronal activity does not significantly affect the proliferation of these cell types in lesions (Supplemental Figure 5, B and D). On the contrary, we observed a 2.83-fold increase in the number of EdU ${ }^{+} \mathrm{Olig} 2^{+}$cells in photostimulated mice compared with controls (Figure $2 \mathrm{E}$ ). To rule out that this increase in OPC proliferation was due to a nonspecific effect of tissue heating by light, we compared the density of proliferative cells in lesions of photostimulated wild-type mice with those of nonphotostimulated Thy1-ChR2-YFP mice. No changes in $\mathrm{EdU}^{+} \mathrm{Olig} 2^{-}$or $\mathrm{EdU}^{+} \mathrm{Olig}^{+}$cell densities were observed between these 2 conditions, showing that the rise of OPC proliferation is due to the increased neuronal activity (Supplemental Figure 4B). Altogether, these results indicate that the activity of demyelinated axons induced by light stimulation specifically enhances the available OPC pool inside the lesion by increasing OPCs' proliferation rate in an activity-dependent manner.

$A$ repeated photostimulation protocol is required to enhance $O L$ differentiation in LPC-induced lesions. During the second week following the LPC injection, a massive differentiation of OPCs onto OLs commonly occurs $(18,19)$. We thus tested whether the increased OPC pool generated by a single photostimulation session at 7 dpi was able to produce more differentiated OLs a week later (Figure 3A). However, OPC and OL densities were unchanged between control and single photostimulated lesions at 14 dpi (Figure 3, B and C), suggesting that the increased OPC pool induced by neuronal activity at 7 dpi was unable to generate mature OLs. To track the proliferative OPC population during the first days following photostimulation, we performed $4 \mathrm{EdU}$ injections per day from $7 \mathrm{dpi}$ to $10 \mathrm{dpi}$ and analyzed the densities of $\mathrm{EdU}^{+} \mathrm{Olig} 2^{+} \mathrm{CC}^{-} \mathrm{OPCs}^{-}$ 
A
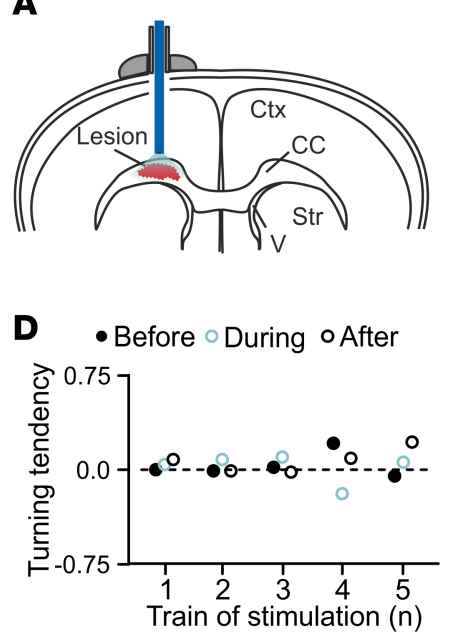

B

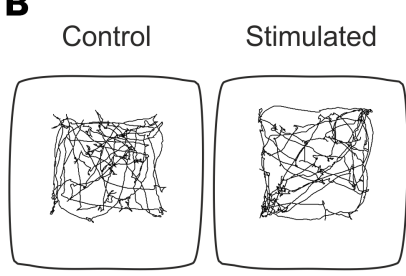

E

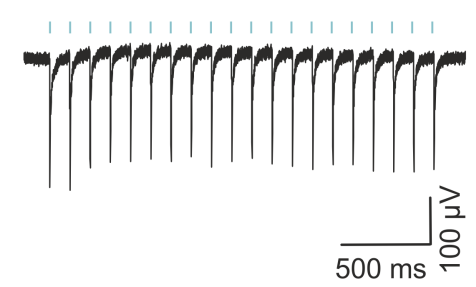

\section{C}
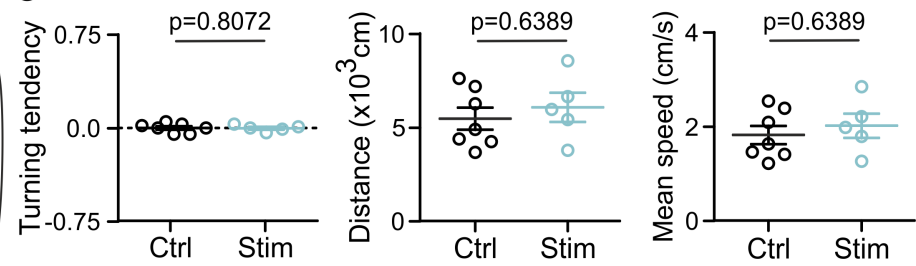

$\mathbf{F}$
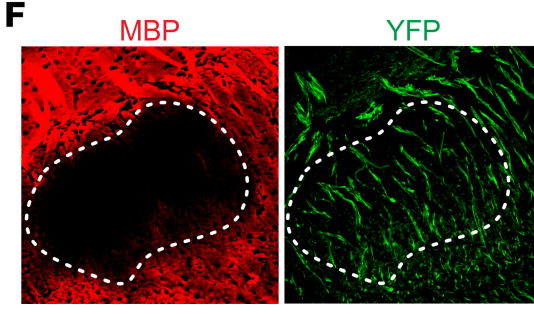

Figure 1. Moderate optogenetic stimulation of ChR2-expressing demyelinated fibers in vivo. (A) Scheme of the optogenetic stimulation of LPC-induced demyelinated lesions in the corpus callosum (CC). The mini-optic fiber is placed above the injected CC. Ctx, cortex; Str, striatum; V, ventricle. (B) Representative open-field trajectory maps ( 5 minutes) of a nonphotostimulated mouse (control) and a photostimulated mouse including the first photostimulation train at $7 \mathrm{dpi}$ (stimulated; 10 -ms light pulses at $20 \mathrm{~Hz}$ for 30 seconds and about 1 megawatt output; $n=7$ controls and $n=5$ stimulated mice). (C) Similar turning tendency (arbitrary units), traveled distance, and mean speed between controls and photostimulated mice over a 50-minute trajectory at $7 \mathrm{dpi}$ for the same mice $(P>0.05$, 2-tailed Mann-Whitney test). Data shown as mean \pm SEM. (D) Turning tendency before, during, and after photostimulation trains (30-second periods) for a single photostimulated mouse at $7 \mathrm{dpi} \mathrm{(} n=7$ control and $n=5$ stimulated mice). (25. AUTHOR: Add units for turning tendency to the y axis in panels $C$ and D.) (E) LFP evoked by photostimulation (blue bars) inside the demyelinated lesion in an anesthetized mouse ( $n=13$ mice). (F) Confocal images of an LPC-induced demyelinated lesion in the CC at $7 \mathrm{dpi}$. The lesion (shown with a dashed line) is recognized by the lack of MBP staining (shown in red). ChR2-expressing fibers are detected by the expression of YFP (shown in green). Scale bar: $100 \mu \mathrm{m}$.

and $\mathrm{EdU}^{+} \mathrm{Olig} 2^{+} \mathrm{CC}^{+} \mathrm{OLs}$ in control and photostimulated mice (see Methods). Despite a large number of $\mathrm{EdU}^{+}$cells stained with this protocol, only a minimal fraction corresponded to $\mathrm{EdU}^{+} \mathrm{Olig} 2^{+} \mathrm{CC}^{+} \mathrm{OLs}$ in both conditions (Supplemental Figure 6). Moreover, no changes were observed for either EdU ${ }^{+}$cells or the total number of OPCs and OLs between photostimulated and nonphotostimulated mice (Supplemental Figure 6, C and D). These results indicate that the OPC pool generated in an activity-dependent manner at 7 dpi was little differentiated into OLs few days later. They also suggest that this pool disappeared within 3 days following the increase in neuronal activity. The rise of OPC density induced by a single photostimulation session at $7 \mathrm{dpi}$ is therefore insufficient to produce long-term modifications in the oligodendroglia population during a week of highly dynamic cellular changes in the demyelinated lesion (18-20).

Considering the transient effect of a single photostimulation session on OPCs, we reasoned that to maintain the new OPC pool induced by neuronal activity at $7 \mathrm{dpi}$ and provoke its development onto mature OLs, it could be necessary to repeat the 3-hour photostimulation protocol every day during the critical period of OPC differentiation, in essence, between 7 dpi and 13 dpi (Figure 3A). In line with this postulate, a repeated photostimulation protocol induced a 1.67-fold increase in the number of mature OLs without causing clear changes in OPC density at 14 dpi (Figure 3, B and C). This OL density increase was not accompanied by a change in the total Olig2 ${ }^{+}$cell density $\left(1112 \pm 98,1039 \pm 92\right.$, and $1285 \pm 67$ cells $/ \mathrm{mm}^{2}$ for control, single, and repeated photostimulation, respectively. $P>0.05$; Kruskal-Wallis statistics $=3.665$; Kruskal-Wallis test followed by Bonferroni's multiple comparison). Thus, a single session of neuronal activity only transiently affects the OPC population in demyelinated lesions, but recurrent neuronal activity for a week is needed to maintain the new OPC pool and promote OPC differentiation, resulting in a significant increase of the population of mature OLs.

Repeated neuronal activity of demyelinated axons in vivo promotes myelin repair and functional recovery. Our repeated photostimulation of demyelinated axons led to an increased pool of OLs inside LPC-induced lesions (Figure 3, B and C). However, an increased number of OLs does not necessarily imply that more newly formed compact myelin will be generated under pathological conditions. To determine whether remyelination is improved after a repeated photostimulation, we performed transmission electron microscopy (EM) analyses of normal-appearing white matter (NAWM), control lesions, and repeatedly photostimulated lesions at $14 \mathrm{dpi}$ (Figure 4A) and quantified remyelinated axons distinguished by their thinner 
A Thy1-ChR2-YFP mouse
Odpi

LPC injection

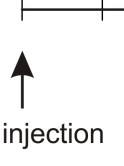

B
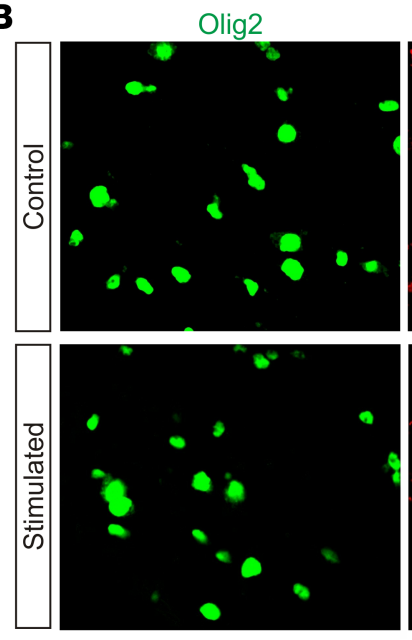

D
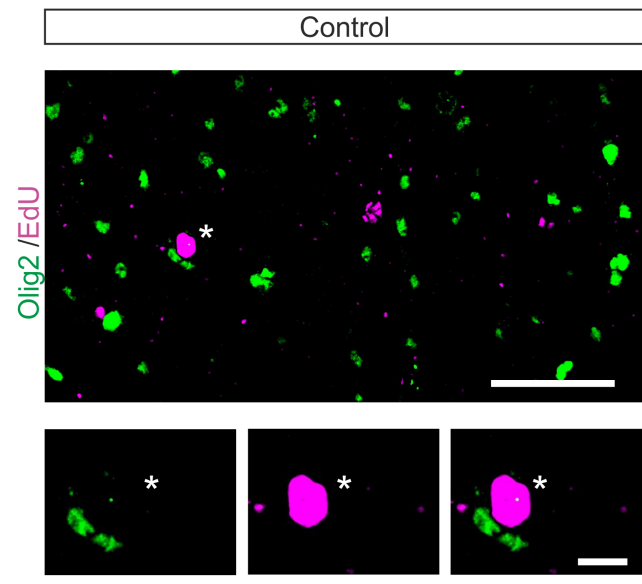
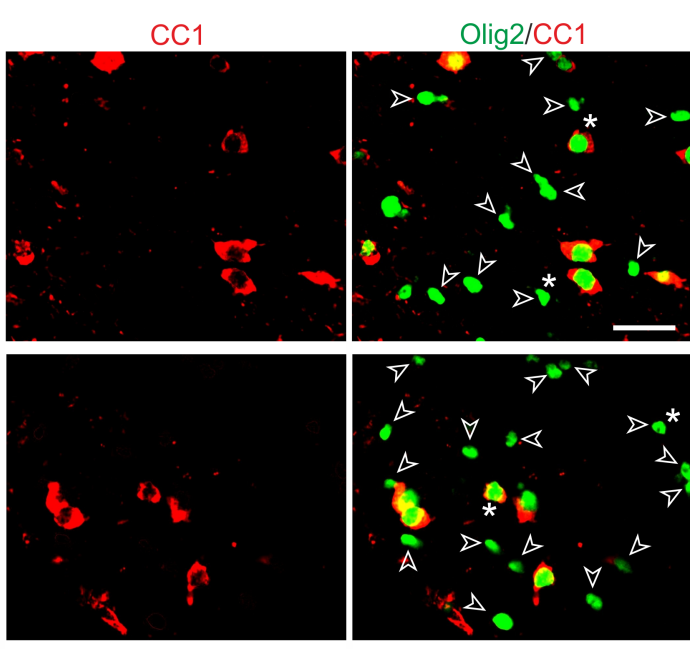

C

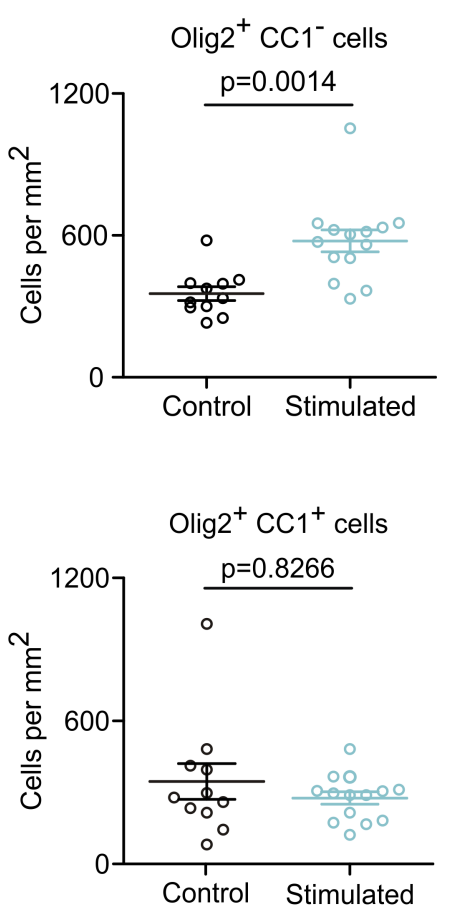

$\mathrm{EdU}^{+}$Olig2 $2^{-}$cells

E

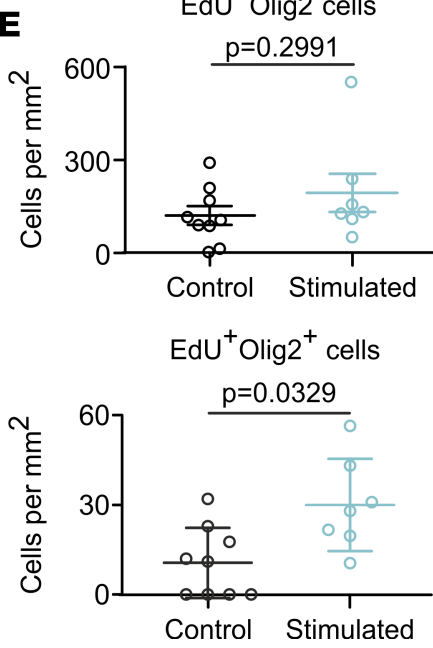

Figure 2. A photostimulation session in vivo increases OPC density in demyelinated lesions. (A) Experimental design at 7 dpi with a 3-hour photostimulation session paradigm. (B) Olig2 (shown in green) and CC1 (shown in red) cells stained at $7 \mathrm{dpi}$ in control LPC-induced lesions (control) and after a photostimulation session (stimulated). Olig2 ${ }^{+} \mathrm{CC} 1^{-}$OPCs are indicated (white arrowheads). Insets: Olig2+CC1- OPCs and Olig2 $2^{+} \mathrm{CC1} 1^{+}$OLs in both conditions. Note that colors are pseudocolors and correspond to Alexa Fluor 405 (405-nm excitation) for Olig2 and Alexa Fluor 633 (633-nm excitation) for CC1. Scale bars: $25 \mu \mathrm{m}, 5 \mu \mathrm{m}$. (C) Density of Olig2 ${ }^{+}$CC1 $1^{-}$OPCs (left) and Olig2 $2^{+} \mathrm{CC} 1^{+}$OLs (right) in control and after a photostimulation session at $7 \mathrm{dpi}$ ( $n=11$ and $n=$ 14 control and stimulated lesions; $U=18.0$ and $U=72.5$, respectively; 2-tailed Mann-Whitney test). (D) Olig2 (green) and 5-ethynyl-2'-deoxyuridine (EdU; magenta) cells stained at $7 \mathrm{dpi}$ in control (control) and photostimulated (stimulated) LPC-induced lesions. Note the presence of EdU+Olig2+ cells (arrowheads) inside the lesions in photostimulated but not control lesions (insets). Note that colors are pseudocolors and correspond to Alexa Fluor 405 (405-nm excitation) for Olig2 and Alexa Fluor 647 (647-nm excitation) for EdU. Scale bars: $50 \mu \mathrm{m}, 10 \mu \mathrm{m}$. (E) Density of EdU+Olig2- (top) and EdU ${ }^{+} \mathrm{Olig2}^{+}$(bottom) cells at $7 \mathrm{dpi}$ ( $n=9$ and $n=7$ control and stimulated lesions; $U=20$ and $U=11$, respectively; 2-tailed Mann-Whitney test). Data shown as mean \pm SEM.

myelin and their decreased myelin optical density profiles (Figure 4B and Supplemental Figure 7, A-C). As expected, axons with thin myelin sheaths, indicative of remyelinated axons (1), were detected in demyelinated lesions at $14 \mathrm{dpi}$, but very few were seen in NAWM (Figure 4, A and B). On the contrary, EM analysis revealed a 2.02-fold increase of remyelinated axons in photostimulated lesions compared with control lesions, indicating that recurrent neuronal activity stimulates myelin regeneration (Figure 4, A and B). 


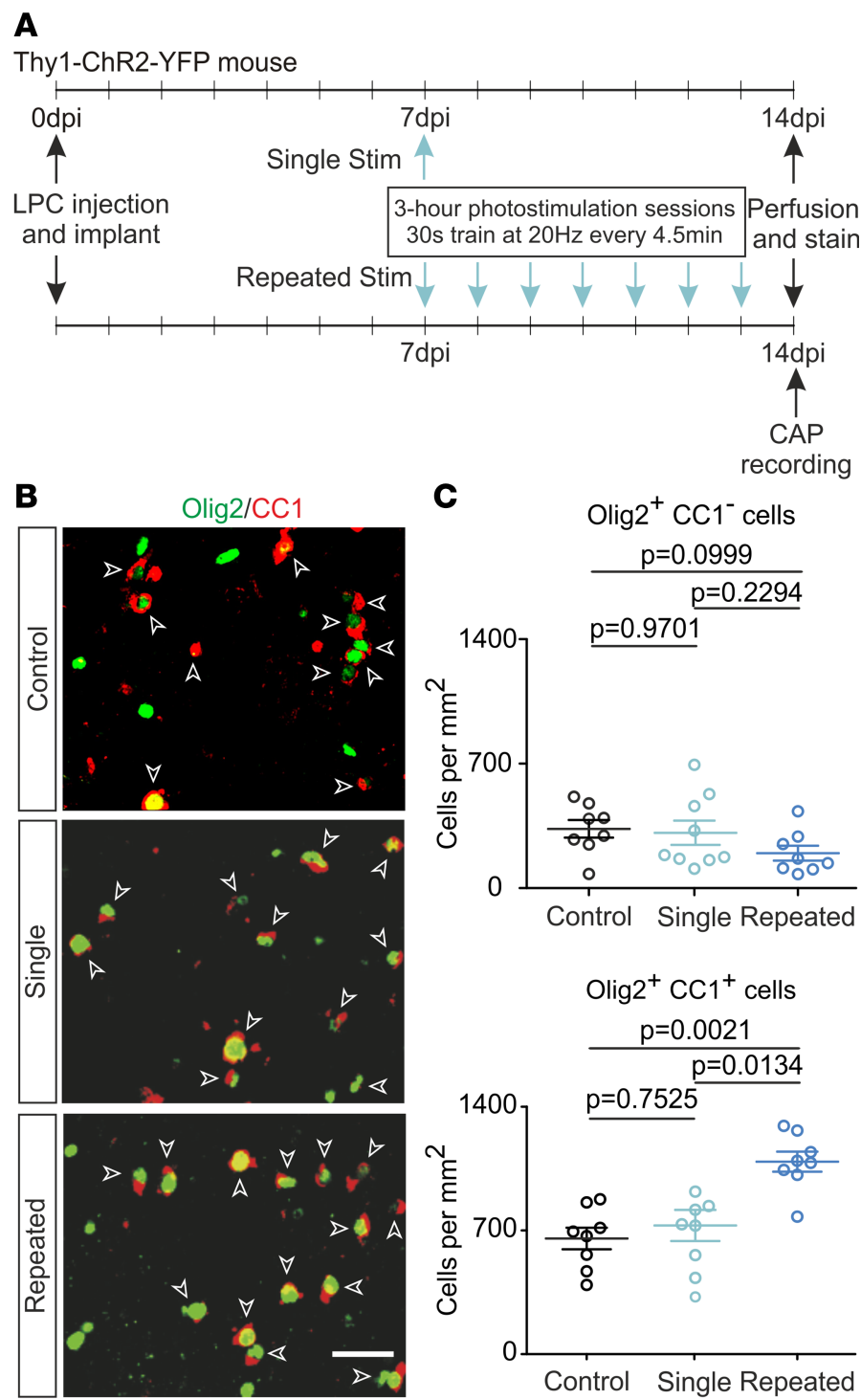

Figure 3. Repeated photostimulation in vivo is required to maintain OPC differentiation in demyelinated lesions. (A) Experimental design with a single and repeated photostimulation paradigm during a week. (B) Olig2 (shown in green) and CC1 (shown in red) cells stained at $14 \mathrm{dpi}$ in control LPC-induced lesions (control), after a single photostimulation session at $7 \mathrm{dpi}$ (single) and after 7 photostimulation sessions over a week (repeated). Mature Olig2 ${ }^{+} \mathrm{CC} 1^{+}$ OLs are indicated (white arrowheads). Note that colors are pseudocolors and correspond to Alexa Fluor 405 (405-nm excitation) for Olig2 and Alexa Fluor 633 (633-nm excitation) for CC1. Scale bar: $25 \mu \mathrm{m}$. (C) Density of Olig2+CC1- OPCs (top) and Olig2 ${ }^{+} \mathrm{CC} 1^{+}$OLs (bottom) in the 3 conditions at $14 \mathrm{dpi}(n=8, n=9$, and $n=8$ lesions for control, single, and repeated protocols, respectively; Kruskal-Wallis statistics $=3.69$ and 11.48 for $\mathrm{Olig2}^{+} \mathrm{CC} 1^{-}$and $\mathrm{Olig2}^{+} \mathrm{CC} 1^{+}$cells, respectively; Kruskal-Wallis test followed by Bonferroni's multiple comparison). Data shown as mean \pm SEM.

Then, we calculated the g-ratio of randomly selected axons, which is an evaluation of the myelin sheath thickness compared with axon diameter, in each condition (Figure 4, C and D). We found that the g-ratio distributions were significantly different among the 3 tested conditions (Figure $4 \mathrm{C}$ ). This difference was not explained by changes in axon size in lesions of control and repeatedly photostimulated mice because axon diameter distributions were not modified with respect to NAWM (Supplemental Figure 7D). However, we observed that the slope of the g-ratio relative to axon diameter after repeated photostimulation was significantly different from that of both NAWM and control lesions (Figure 4D), suggesting that the increased remyelination resulting from repeated photostimulation did not equally occur in demyelinated axons of different size. In fact, for axons displaying a diameter smaller than $0.793 \mu \mathrm{m}$, which corresponded to the intersection of linear fits for control lesions and repeated photostimulation conditions, the myelin thickness 
A

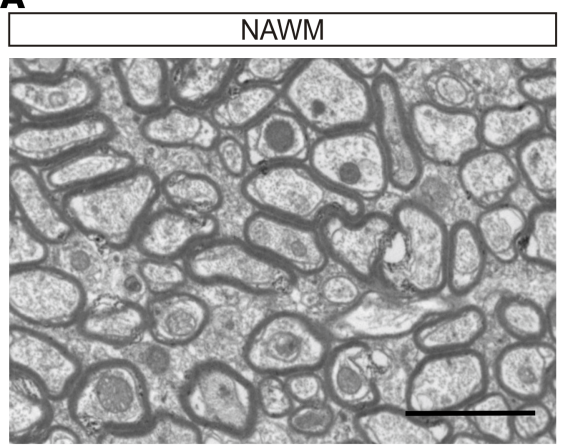

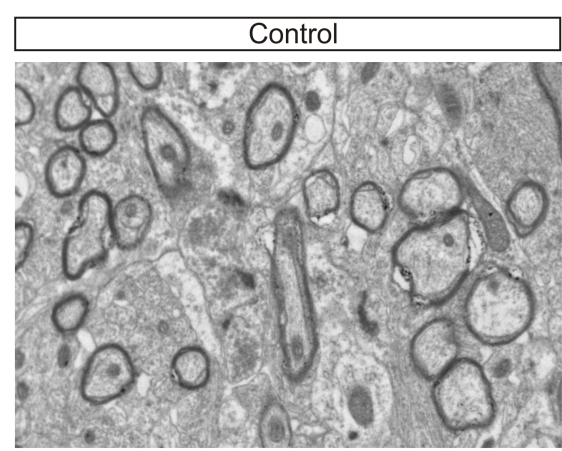

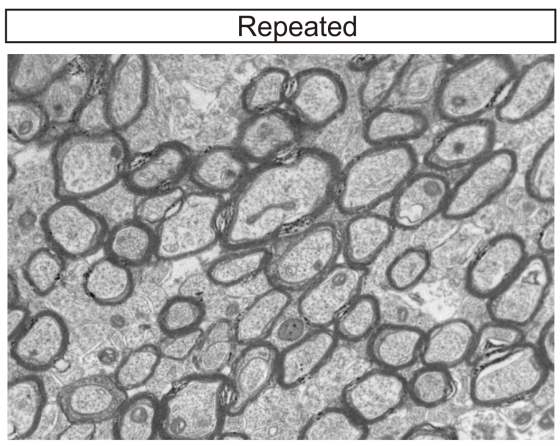

B

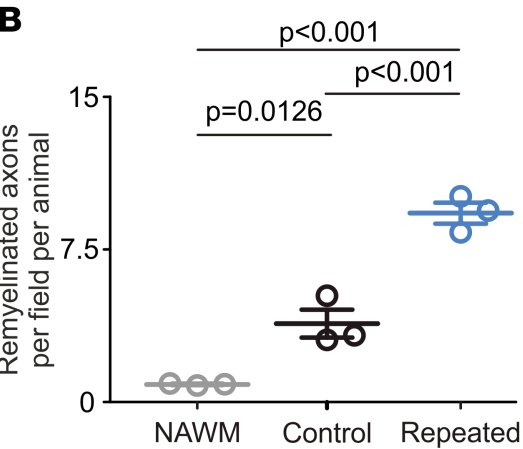

C

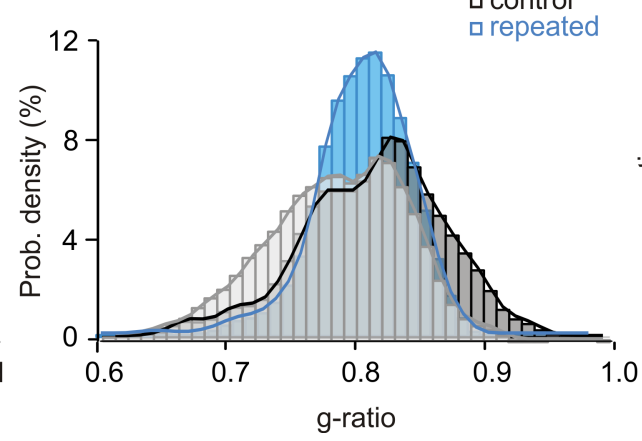

D

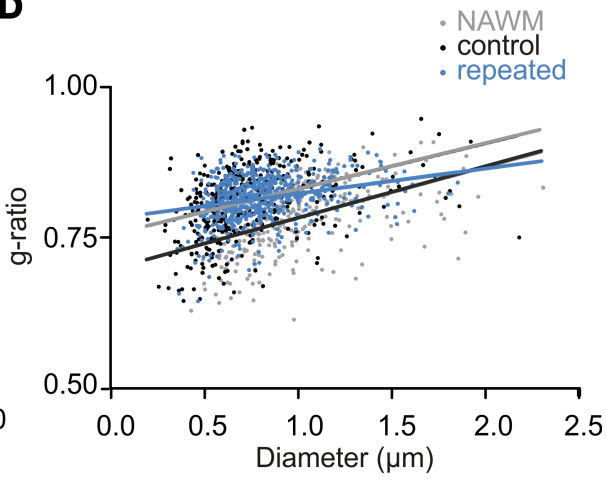

Figure 4. Repeated photostimulation in vivo enhances remyelination. (A) Electron micrographs of transverse sections from nondemyelinated CC (NAWM), control LPC-induced lesions (control), and repeatedly photostimulated LPC-induced lesions (repeated) at $14 \mathrm{dpi}$ ( $n=3 \mathrm{mice}$ per condition). Scale bar: $2 \mu \mathrm{m}$. (B) Dot plot of the average number of remyelinated axons per field per animal for NAWM, control, and repeated LPC-induced lesions at $14 \mathrm{dpi}$ ( $n=3$ mice per condition; $F=73.96$; 1 -way ANOVA test followed by Bonferroni's multiple comparison). Data shown as mean \pm SEM. (C) Distribution of g-ratio values per condition $(n=302, n=545$, and $n=812$ measured axons for each condition, respectively; $D=0.144, P=0.001$ for NAWM vs. control; $D=0.238, P=0.001$ for NAWM vs. repeated; $D=0.102, P=0.042$ for control vs. repeated; Kolmogorov-Smirnov test). (D) Scatter plot of the same g-ratio values as a measure of myelin thickness for NAWM (shown in gray), control (shown in black), and repeated LPC-induced lesions (shown in blue) (comparison of slopes: $F=9.8643, P=0.687$ for NAWM vs. control, and $P<0.0001$ for both NAWM vs. repeated and control vs. repeated; 1-way ANOVA test followed by Bonferroni's multiple comparison).

was almost identical in control lesions compared to photostimulated mice but different from NAWM (Figure 4D; g-ratio: $0.741 \pm 0.003,0.753 \pm 0.004$, and $0.766 \pm 0.005$ for NAWM, control lesions, and repeated photostimulation, respectively. Kruskal-Wallis statistics $=27.79 ; P=0.411$ for repeated vs. control; $P<$ 0.0001 for NAWM vs. control; and $P=0.0001$ for repeated vs. NAWM. Kruskal-Wallis test followed by Bonferroni's multiple comparison). On the contrary, for axons displaying a diameter larger than $0.793 \mu \mathrm{m}$, the g-ratios of repeated photostimulation and NAWM conditions were similar but significantly smaller compared with control (Figure 4D; g-ratio: $0.830 \pm 0.002,0.843 \pm 0.003$, and $0.830 \pm 0.001$ for NAWM, control lesions, and repeated photostimulation, respectively. Kruskal-Wallis statistics $=43.73 ; P=1.0$ for repeated vs. NAWM; and $P<0.0001$ for both repeated vs. control and NAWM vs. control. Kruskal-Wallis test followed by Bonferroni's multiple comparison). These findings indicate that the enhanced remyelination promoted by repeated neuronal activity is biased toward larger diameter axons. However, it is not biased toward ChR2-expressing axons because the proportion of myelinated $\mathrm{YFP}^{+}$axon segments with respect to the total myelinated axon segments did not change between control and photostimulated mice (Supplemental Figure 7, E and F).

To assess whether the activity-dependent enhanced remyelination was accompanied by a functional improvement of conduction in photostimulated mice, we recorded evoked compound action potentials (CAPs) in acute coronal slices of corpus callosum at $14 \mathrm{dpi}$ after performing in vivo experiments (Figure 3A). The demyelinated area was recognized as a brighter region surrounding white matter (Supplemental Figure 2B). In the normal corpus callosum (NAWM), CAPs were characterized by the presence of fast and slow components corresponding respectively to myelinated and unmyelinated axon responses (21) (Figure 5A). As expected in control demyelinated lesions, the fast component was highly reduced or disappeared (Figure 5A). 
However, the increase in remyelination after repeated photostimulation induced a recovery of the fast component, which had the same conduction velocity as NAWM (Figure 5, A and B). Moreover, the normalized AUC of the fast component was smaller in control lesions but reached normal values after repeated photostimulation (Figure 5B). Finally, comparison of normalized CAP amplitudes revealed that the fast component was significantly reduced in control lesions with respect to NAWM but rescued in photostimulated mice (Figure 5B). Altogether, these experiments indicate an extensive ultrastructural and functional recovery of demyelinated fibers upon repeated photostimulation of demyelinated axons in vivo.

\section{Discussion}

This report demonstrates that moderate and controlled neuronal activity in vivo increases the pool of mature OLs, enhances remyelination, and restores action potential conduction in demyelinated lesions. We also show that the recurrence of lesion stimulation constitutes an important factor to take into account. Although a single episode of activity has only a transient effect on the OPC population, recurrent activity promotes a robust increase in OL density and enhances remyelination. Therefore, neuronal activity is able to enhance OPC differentiation and myelin synthesis in an altered environment such as a demyelinated lesion. Remyelinated injured axons, in turn, undergo a significant functional recovery.

Recent studies have demonstrated that neuronal activity promotes OL differentiation in the healthy brain, but the cellular mechanisms responsible for this effect are not completely clear. A transient 30-minute photostimulation of ChR2-expressing motor neurons was sufficient to increase OPC proliferation followed by a later more modest rise in the OL population in the neocortex and corpus callosum (6). On the contrary, a single DREADD-mediated (designer receptors activated by designer drugs) neuronal stimulation did not cause modifications in the number of cycling OPCs in the corpus callosum at the level of the somatosensory cortex (9). Sustained stimulation for 7 days using a DREADD approach was needed to induce OPC proliferation and a subsequent differentiation of newly divided OPCs into myelinating OLs (9). Although understanding the causes of the different cellular responses of OL lineage cells to neuronal activity is not straightforward, substantial differences exist among studies. In Gibson et al. (6), the optogenetic stimulation specifically targeted layer V pyramidal neurons, whereas in Nagy et al. (14) and Mitew et al. (9), the number and selectivity of stimulated fibers were probably more heterogeneous because the fibers recruited during electrical stimulation and the efficiency of in utero electroporation, respectively, are variable. Finally, the dynamics of both OPCs and OLs can be easily manipulated by experience and injury in young mice $(22,23)$. Although experience-dependent enhancement of OL production and myelination in the mature cortex also occurs (24), the activity-dependent OPC plasticity declines with age (9). Therefore, different preparations, animal ages, and stimulation paradigms including various intensities, frequencies, and durations probably cause different effects (14). In this context of complex cellular responses, it is unknown whether the activity of demyelinated axons in lesions, where environmental factors causing the damage radically change the conditions, can have a positive effect on remyelination. We show that a single photostimulation session at $7 \mathrm{dp}$ in the adult induces a rapid increase in the OPC population. As in Gibson et al. (6), this rapid rise in OPC density is probably caused by an increase in the proliferative fraction. However, the OPC density increase at 7 dpi was not associated with an increased number of OPCs and mature OLs 3 days or 1 week after a single episode of increased neuronal activity. This transient rise in the OPC pool suggests that, in the absence of increased activity, the survival of a large number of OPCs inside the lesion is compromised. Although this apparently homeostatic response needs further investigation, our findings clearly show that recurrent but moderate neuronal activity during a week was needed to maintain the OPC pool and induce its differentiation into myelinating OLs. Thus, recurrent photostimulation probably shifts the balance from OPC proliferation toward more differentiation. This process could also act in conjunction with an activity-dependent prosurvival effect $(22,24)$. Collectively, these data provide evidence for the existence of different responses of OL lineage cells to neuronal activity that may vary according to the number and type of stimulated fibers, the frequency and patterns of stimulation, the neurotransmitters' and signaling molecules' release during neuronal activity, the age of the animals, and physiological versus pathological conditions.

The concept of activity-driven adaptive myelination represents an important recent advance in our understanding of the myelination process (25). However, the molecular mechanisms linking neuronal activity with oligodendroglia development and myelin production remain poorly understood. Because axon-oligodendroglia interactions induce $\operatorname{OPC}$ differentiation $(10,26)$ and myelin formation $(8,10,27)$, it is conceivable that neuronal activity directly triggers molecular changes in OPCs and OLs. These glial 
A
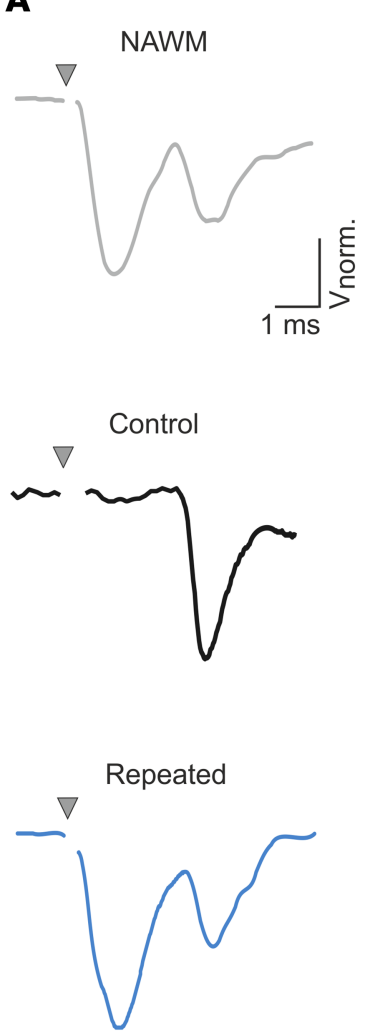

B
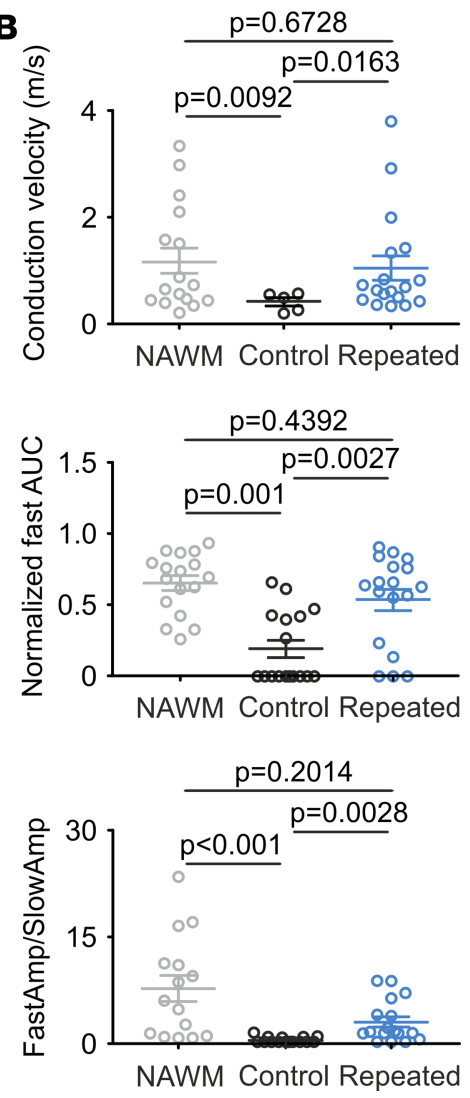

Figure 5. Repeated photostimulation in vivo promotes functional recovery in demyelinated lesions. (A) Representative CAPs for NAWM, control, and repeated photostimulated lesions at $14 \mathrm{dpi}$. CAPs were obtained after subtracting average traces before and after application of $1 \mu \mathrm{M}$ TTX (19). Note that the first peak corresponding to myelinated fibers disappeared in control LPC-induced lesions but was rescued after repeated stimulation. The time of stimulation is indicated (gray arrowheads). The stimulation artifacts were blanked for visibility. (B) Dot plots of conduction velocity (top), normalized area under the curve (AUC; middle) for the fast component, and amplitude ratio between fast and slow components of CAPs (fastAmp/slowAmp; bottom). Note that only 5 out of 17 recordings in control LPC-induced lesions had a fast component ( $n=17$ recordings for NAWM and control, and $n=18$ recordings for repeated; $n=3$ mice per condition; Welch's $t$-corrected test value $[\mathrm{W}-\mathrm{t}]=0.43$ and df $=41$, W-t st. $=2.94$, and df $=17, \mathrm{~W}$-t st. $=2.62$, and df $=20$, respectively, for conduction velocity; Welch's $t$-corrected test; Kruskal-Wallis statistics $=18.40$ for normalized AUC, and Kruskal-Wallis statistics $=20.85$ for fastAmp/slowAmp; Kruskal-Wallis test followed by Bonferroni's multiple comparison). Data shown as mean $\pm \mathrm{SEM}$.

cell types express different synaptic and extrasynaptic receptors for neurotransmitters according to their developmental state (28). An interesting possibility is therefore that neurons displaying different levels of activity or cell identities activate distinct neurotransmitter receptors on oligodendroglia that, in turn, modulate the expression of transcription factors and epigenetic regulators involved in OPC maturation and OL myelin production. Other factors released by neurons, such as brain-derived neurotrophic factor, neuregulin, or fractalkine, may also be released in an activity-dependent manner to promote OPC differentiation and myelination (29-31). Alternatively, astrocytes and microglia could indirectly contribute to activity-driven adaptive myelination. For instance, enhanced neuronal activity in cell cultures promotes myelination through ATP-dependent secretion of leukemia inhibitory factor from astrocytes (32). In addition, astrocytes and microglia release different soluble factors that, in demyelinated conditions, have either detrimental or beneficial effects on OPC fate and myelin repair (20,33). Further investigations are needed to understand whether neuronal activity influences the release of these factors and mediates glia-oligodendroglia interactions in normal and pathological conditions.

Whether myelin sheaths on remyelinated axons also display activity-dependent plasticity was an open question (1). Our study demonstrates that recurrent stimulation of demyelinated fibers not only modifies oligodendroglia behavior inside lesions but also induces an impressive improvement in functional remyelination. Hence, the process of myelin repair in demyelinated lesions is also plastic, although differences between 
physiological and pathological conditions exist. During development, more neuronal activity results in thicker myelin sheaths with a global decreased g-ratio $(6,9)$. Following demyelination, however, we did not observe thicker remyelinated axons after repeated photostimulation compared with NAWM. We found that recurrent neuronal activity induces a 2 -fold increase in the number of axons undergoing remyelination as revealed by their thinner myelin. This increase probably represents an underestimation of the number of remyelinated axons if we considered that, in these counts, we did not consider those axons displaying near-normal myelin in the lesion. Indeed, further EM analysis performed in randomly selected axons showed that the activity-dependent myelin formation appears biased toward axons with diameters larger than $0.793 \mu \mathrm{m}$, for which the average g-ratio reached the value obtained for NAWM. In addition, the g-ratio distribution changed from being bimodal in both control lesions and NAWM to being a single Gaussian after repeated photostimulation (Figure $4 \mathrm{C}$ ), suggesting that the evoked activity of layer $\mathrm{V}$ pyramidal axons induces a preferential phenotype of axon remyelination. Although we observed an effect biased toward larger axons, we cannot totally rule out that an enhanced activity-dependent remyelination occurs in both small and large axons, but with larger axons displaying a proportionally thicker myelin sheath and, thus, a smaller g-ratio. Indeed, we did not observe a preferential myelination of ChR2-expressing $\mathrm{YFP}^{+}$axons, which largely belongs to layer V pyramidal cells known to display large-diameter axons (16). In any case, if myelin were thicker in larger diameter axons, this would imply that remyelination of those axons was of better quality than that of smaller ones, probably because many of them were ChR2-positive and thus activated by light. Moreover, we observed that repeated photostimulation was accompanied by a full recovery of the conduction velocity of CAPs measured inside the lesion (Figure 5). This full recovery is in agreement with the fact that CAP measurements are biased to account mostly for the activity of larger diameter axons $(34,35)$. Indeed, CAP recordings are a robust but also an inhomogeneous averaged way to measure the conduction of a diverse axon population (35). Nevertheless, our data show that activity extensively improves remyelination and conduction, a repairing process that should be beneficial to protect axons from degeneration (36).

It was recently shown that pharmacological therapies with drugs known to stimulate OL differentiation constitute a possible approach in demyelinating diseases (37). This finding emphasizes the importance of understanding the biological basis for a successful endogenous remyelination, a necessary condition to identify effective strategies enhancing myelin repair. An important first step to achieve this goal is to know the intrinsic and extrinsic factors that modify oligodendroglia genesis and fate as well as the sequence of events in which these factors act (38). Our study is the first to our knowledge to demonstrate a strong remyelinating effect of neuronal activity in vivo and establish the conditions in which this activity is highly efficient in pathological conditions. Our findings provide the proof of concept for new clinical trials aiming at testing the therapeutic efficacy of either electrical or chemical neuromodulation. Clinical studies have already explored neuromodulation as a possible treatment for demyelinating diseases, such as MS (39). Thalamic deep brain stimulation in MS patients has shown improvements in the typically associated tremor $(40,41)$ and ameliorates the neuropathic pain of MS-induced trigeminal neuralgia (42). Likewise, repetitive transcranial magnetic stimulation of the motor cortex improved some of the associated symptoms in MS patients, such as spasticity and cerebellar dysfunction $(43,44,45)$. However, despite the potential beneficial effects of these approaches, available data are still sparse and sometimes contradictory $(39,46)$. Our results establish fundamental cellular principles of how the activity of demyelinated axons, when they still have the capacity to drive action potentials, leads to changes in OL lineage cell dynamics, promoting OL differentiation and improving functional remyelination in the damaged tissue. Our work supports the notion that activity-dependent myelin repair of the CNS can be achieved after a demyelinating injury and opens new, promising avenues for early therapeutic intervention to enhance myelin regeneration in demyelinating diseases, such as MS.

\section{Methods}

Animals and surgery. Experiments were performed with heterozygous male and female C57BL/6 Thy1ChR2-YFP-transgenic adult mice (8 to 9 weeks old) obtained from The Jackson Laboratory (stock 007612) (15) and backcrossed for more than 10 generations. A few experiments were also performed using male and female C57BL/ 6 wild-type mice of the same age.

To induce a focal demyelinating lesion, mice were deeply anesthetized with ketamine/xylazine $(0.1 / 0.01 \mathrm{mg} / \mathrm{g}$, i.p.) and fixed in a stereotaxic frame (Kopf Instruments). Ophthalmic Dexpanthenol gel (Chauvin Bausch \& Lomb GmbH) was applied to the eyes to prevent dehydration. The skin above the skull was disinfected and incised. Then, a hole was stereotaxically drilled on the motor cortex area to allow a 
glass pipette connected to a Hamilton syringe to be lowered into the corpus callosum to inject $2 \mu 1$ of LPC (MilliporeSigma, $1 \%$ in PBS), as we previously described (coordinates: $1 \mathrm{~mm}$ lateral to midline, $1.5 \mathrm{~mm}$ anterior to the bregma, and 1.8-mm depth from the brain surface) (19).

For optogenetic stimulation, the same hole used for LPC injections was accessed to implant a cannula accommodating a mini-optic fiber (cannula: $1.25 \mathrm{~mm}$; fiber: $200-\mu \mathrm{m}$ diameter, numerical aperture = 0.66; Prizmatix Ltd.). This manipulation was performed 10 minutes after LPC injections during the same surgery. The mini-optic fiber was stereotaxically lowered into deep layers of the motor cortex, above the injected corpus callosum (fiber: 1.4-mm protrusion). Then, the optogenetic implant was fixed to the skull with dental cement (Unifast Trad Ivory 339104, Gc America Inc.) (Figure 1A). Finally, the skin was sutured (Mersilene, EH7147H, Ethicon) around the implant. Mice were recovered from anesthesia in an environment at $37^{\circ} \mathrm{C}$ before they were returned to their home cages.

Photostimulation and LFP recordings in vivo. The day of the photostimulation, the mini-optic fiber was connected to a 460-nm LED source through a fiber optic patch cord (Prizmatix). The LPC-induced demyelinated area was photostimulated in freely moving mice with a 3-hour protocol composed of 36 light trains of 30 seconds delivered at $20 \mathrm{~Hz}(10 \mathrm{~ms}$ on/40 ms off pulses) and separated by a resting period of 4.5 minutes. The power at the tip of each mini-optic fiber was systematically checked with an optical power meter (PM100D coupled with sensor S120C, Thorlabs) before surgery and set at about 1 milliwatt (mW) the day of the experiment. Mice were randomly allocated to 3 experimental groups: (a) a single 3-hour photostimulation session at $7 \mathrm{dpi}$ (single), (b) 3-hour photostimulation session repeated every day from 7 to $13 \mathrm{dpi}$ (repeated), and (c) no photostimulation session but subjected to the same surgical procedures and connected to the fiber optic patch cord (control).

To confirm that demyelinated fibers inside LPC-induced lesions displayed neuronal activity upon in vivo photostimulation, a set of experiments was performed with a custom-made optrode built by attaching to the mini-optic fiber a recording electrode whose tip reached the core of the demyelinated lesion (Ni/Ag wire, $50-\mu \mathrm{m}$ diameter; about $1.6 \mathrm{~mm}$ from the surface). The optrode was implanted and fixed to the mouse skull as described above. A pin connector was also fixed to the skull surface at the contralateral hemisphere and used as a ground for in vivo recordings (coordinates: $1 \mathrm{~mm}$ caudal and lateral to $\lambda$ ). LFP recordings were obtained in anesthetized mice (ketamine/xylazine $0.1 / 0.01 \mathrm{mg} / \mathrm{g}$ ) after connecting the optrode to both the preamplifier and the 460-nm LED source system. Light trains from 1 to 30 seconds delivered at 20 $\mathrm{Hz}$ were applied ( $1 \mathrm{~mW}$; 10-ms light pulses), while light-evoked LFPs were recorded in current clamp (current $=0$ ) voltage follower mode. To confirm that the activation of ChR2 $2^{+}$fibers induced LFPs in lesions, the same experiment was performed in wild-type mice (negative controls). All electrophysiological data of this study were acquired at $20 \mathrm{kHz}$ by using a MultiClamp 700B amplifier, filtered at $4 \mathrm{kHz}$, digitized with a Digidata 1440A, and analyzed offline with Pclamp10.1 software (all Molecular Devices) and IgorPro 6.0 software (Wavemetrics).

Open-field behavior. Freely moving mice were video-recorded during photostimulation by using a CMOS camera (DFK22AUC03, Imaging Source) positioned above a standard open-field box. Mouse behavior was monitored and analyzed by Smart video tracking software (version 3.05.05, PanLab). Light pulse delivery was coordinated with the video acquisition by a TTL pulse controller (Pulser software, Prizmatix). To investigate motor behavior changes during photostimulation, we compared the turning tendency, the mean speed (with or without resting time), and the total traveled distance for 50-minute video recordings between photostimulated and control groups. We also analyzed these parameters before (30 seconds), during (30 seconds), and after (30 seconds) a train (for 10 trains).

Electrophysiology in acute brain slices. Acute coronal slices of LPC-injected corpus callosum (300 $\mu \mathrm{m})$ were obtained from Thy1-ChR2-YFP-transgenic adult mice (8 to 9 weeks old) as previously described (19). Slices were placed in a recording chamber perfused with a standard extracellular solution ( $3 \mathrm{ml} / \mathrm{min})$ containing (in mM): $126 \mathrm{NaCl}, 2.5 \mathrm{KCl}, 1.25 \mathrm{NaH}_{2} \mathrm{PO}_{4}, 26 \mathrm{NaHCO}_{3}, 20$ glucose, 5 pyruvate, $3 \mathrm{CaCl}_{2}$, and $1 \mathrm{MgCl}_{2}$ (pH: 7.43; 95\% $\mathrm{O}_{2}, 5 \% \mathrm{CO}_{2}$ ). For whole-cell recordings, layer V cortical YFP ${ }^{+}$pyramidal neurons were visualized by fluorescence and differential interference contrast (DIC) videomicroscopy and recorded with a patch pipette using an intracellular solution containing (in $\mathrm{mM}$ ): $120 \mathrm{~K}$-gluconate, $5 \mathrm{NaCl}, 3 \mathrm{MgCl}_{2}$, 0.2 EGTA, 10 HEPES, 0.3 Na-GTP, 4 Na-ATP, and 10 Na-phosphocreatine (pH 7.3, 295 mOsm). Lightevoked action potential discharges of $\mathrm{YFP}^{+}$pyramidal neurons were elicited by light trains of different duration and delivered through a fiber optic placed above the patch pipette (fiber: $200-\mu \mathrm{m}$ diameter; numerical aperture: 0.53; power: $1 \mathrm{~mW}$; Prizmatix). 
For recordings of CAPs, demyelinated lesions were recognized under the microscope as a brighter area in the corpus callosum (Supplemental Figure 2B). CAPs were obtained by stimulating white matter fibers with a monopolar electrode placed outside the lesion, while the recording electrode (glass pipette) was placed in the lesion core ( $60 \mathrm{~V}$; $100-\mu$ s stimulations; Iso-Stim $01 \mathrm{D}$, npi electronic $\mathrm{GmbH}$ ). To calculate the conduction velocity, stimulation pulses were delivered at 2 different positions with respect to the recording electrode. The amplitudes of fast and slow components, the AUC, and the conduction velocity were analyzed using acute slices of repeatedly stimulated and control demyelinated mice, as we previously described (21). CAPs were also measured in NAWM for comparison. For a more reliable comparison, the 3 conditions (NAWM, control, repeated) were tested the same day with the same stimulation and recording electrodes ( $n=3$ mice per condition).

Immunostainings, EdU proliferation assays, and counts. Animals were perfused intracardially with PBS followed by $0.15 \mathrm{M}$ phosphate buffer (PB) containing 4\% paraformaldehyde (PFA, Electron Microscopy Sciences), $\mathrm{pH}$ 7.4. For experiments at $7 \mathrm{dpi}$, mice were perfused 1 hour after the end of the 3-hour protocol. For experiments at $14 \mathrm{dpi}$, mice were perfused 1 day after the end of the last 3-hour protocol. After perfusion, brains were kept 1 hour in PFA and stored in PBS at $4^{\circ} \mathrm{C}$ before cutting. Sagittal vibratome slices $(120 \mu \mathrm{m})$ were prepared in $\mathrm{PBS}$ at $4^{\circ} \mathrm{C}$ and permeabilized with $1 \%$ Triton X-100 and 4\% normal goat serum (NGS) for 2 hours.

Immunostainings against Olig2, CC1, and MBP were performed with rabbit anti-Olig2 (1:400, AB9610, MilliporeSigma), mouse monoclonal anti-CC1 (1:100, OP80, Calbiochem), and rat monoclonal anti-MBP (1:100, AB7349, Abcam) diluted in 0.2\% Triton X-100 and 4\% NGS. Slices were first incubated with primary anti-Olig2 and anti-CC1 antibodies for 3 nights and exposed for 2 hours at room temperature (RT) with secondary antibodies coupled to Alexa Fluor 405 and Alexa Fluor 633, respectively (1:500, Thermo Fisher Scientific). Next, the same slices were incubated with the primary anti-MBP antibody 1 more night and exposed for 2 hours at RT with a secondary antibody coupled to Alexa Fluor 555 (1:500, Thermo Fisher Scientific). Negative controls were treated by omitting all primary antibodies or by incubating a primary antibody with a secondary antibody against an omitted primary antibody.

To label cells in the S phase of the cell cycle, we performed i.p. EdU injections at 7 dpi (C10340, Thermo Fisher Scientific). In a first protocol, mice were injected 10 minutes before and after the 3-hour protocol (50 mg/kg per injection) at $7 \mathrm{dpi}$ and perfused 1 hour later. Slices of EdU-treated mice were first immunostained with either rabbit anti-Olig2 and rat anti-MBP or rat anti-MBP, rabbit anti-IBA1 (1:1,000, 019-19741, Wako), and mouse anti-GFAP (1:100, G3893, MilliporeSigma). In a second protocol, mice were injected 4 times per day for 3 hours from $7 \mathrm{dpi}$ to $10 \mathrm{dpi}(20 \mathrm{mg} / \mathrm{kg}$ per injection) and perfused 1 hour after the last injection. In this case, slices of EdU-treated mice were immunostained with rabbit anti-Olig2, mouse anti-CC1, and rat anti-MBP as described above. EdU was exposed after immunostainings by using the Clik-iT EdU Alexa Fluor 647 (C10340, Thermo Fisher Scientific).

Confocal images were mainly acquired using a $\times 20(\mathrm{NA}=0.8 ; 1.5-\mu \mathrm{m}$ z-step $)$ and $\times 63(\mathrm{NA}=1.4$; $0.75-\mu \mathrm{m} z$-step) oil objective (NA $=0.8$ and 1.4 , respectively) with an LSM 710 confocal microscope (Zeiss) and processed using the NIH's ImageJ software as previously described (47). For the analysis of the proportion of myelinated $\mathrm{YFP}^{+}$fibers in control and photostimulated mice, we used a $\times 93$ glycerol objective (NA $=1.3 ; 0.35-\mu \mathrm{m}$ z-step) mounted in an SP8 Leica confocal microscope and quantified myelinated fiber segments as we previously described (47) in images of $62.5 \times 62.5 \mu \mathrm{m}^{2}$ of $25 \mathrm{Z}$-sections. LPC-induced lesions were identified by the lack of MBP expression with a $\times 20$ objective and imaged for cell counts with a $\times 63$ oil objective. The lesion area was identified by the lack of MBP expression and delimited manually before cell counts. It is noteworthy that some degree of remyelination had already occurred at $14 \mathrm{dpi}$, but the MBP staining was still sufficiently lower inside the lesion compared with a normal corpus callosum to delineate the lesion. Cell densities were obtained by counting OPCs and OLs with the ROI Manager Tool (ImageJ) and by dividing the number of cells by the defined demyelinated area.

Transmission EM. For ultrastructural visualization of myelinated axons, mice at 14 dpi were perfused with $5 \%$ glutaraldehyde and 4\% PFA in PB 0.1 M, pH 7.4. Brains were removed and immersed in the same solution for 1 hour. After rinsing brains with $\mathrm{PB}, 100-\mu \mathrm{m}$ sagittal sections spanning the entire lesion area were obtained with a vibratome (Leica VT1000S) and subsequently incubated in $2 \% \mathrm{OsO}_{4}$ for 1 hour. Then, sections were rinsed in distilled water and contrasted with uranyl acetate $5 \%$ for 30 minutes. Samples were dehydrated in a graded series of ethanol and cleared in acetone. Sections were then embedded in epoxy resin (Agar Scientific) and incubated at $56^{\circ} \mathrm{C}$ for 48 hours to allow for polymerization. Once polymerized, blocks were observed at the dissection microscope, and the lesion area was manually microdissected. Dissected blocks were first cut 
into $1-\mu \mathrm{m}$ semithin sections with an ultramicrotome (UC7, Leica). Sections were picked up every $1 \mu \mathrm{m}$ and stained with toluidine blue. Semithin sectioning was performed until the lesion area was observed. Then, serial ultrathin sections (70 nm thick) were collected onto copper grids (about 3-4 sections per grid) and visualized at the transmission electronic microscope (Hitachi HT7700). Electron micrographs from the lesion areas were taken at $\times 18,000$ magnification, using an integrated AMT XR41-B camera $(2,048 \times 2,048$ pixels $)$. Measurements were made blind using the NIH's ImageJ for control and repeatedly photostimulated lesions ( $n$ $=3$ and $n=5$, respectively). We compared these conditions with NAWM corresponding to a nonlesioned corpus callosum of mice injected with LPC but not photostimulated. G-ratio was calculated by dividing the axon diameter by the total diameter of the axon overlaying the myelin sheath. Two perpendicular measurements of the largest and shortest diameters per axon were averaged to calculate the g-ratio. To estimate the number of remyelinated axons in each condition, images were divided into 4 fields, and the number of remyelinated axons per field was counted. We considered as remyelinated axons those displaying a low optical density and a g-ratio greater than 0.82 (Supplemental Figure 7). This value corresponds to the second peak of the g-ratio distribution of control lesions (Figure 4C). To compare the distributions of g-ratio values among conditions, we calculated the g-ratio of at least 300 randomly selected axons per condition.

Statistics. All data were expressed as mean \pm SEM. A $P$ value less than 0.05 was considered significant. Because some data sets contained tied values, each data group was subjected to a D'Agostino-Pearson normality test rather than a Shapiro-Wilk test. According to the data structure, 2 group comparisons were performed using 2-tailed, unpaired Student's $t$ test or Mann-Whitney test. When data did not fit the assumption of equal variances, a Welch's corrected $t$ test was applied. Bonferroni's multiple comparisons were used as the post hoc test following 1-way and 2-way ANOVA or the nonparametric Kruskal-Wallis test. Data set distributions were compared by using the Kolmogorov-Smirnov distribution test. Statistics and plotting were performed using Prism 5.00 (GraphPad Software Inc.), R package (https://www.r-project.org/), and Curve Expert 1.4 (Microsoft Corporation) software.

Study approval. All experiments followed European Union and institutional guidelines for the care and use of laboratory animals and were approved by both the French ethical committee for animal care of University Paris Descartes (Paris, France) and the Ministry of National Education and Research (authorization 02015010514019852).

\section{Author contributions}

FCO and $\mathrm{CH}$ conducted optogenetic, electrophysiological, and behavioral experiments; immunostainings; and data analysis. MG performed EM experiments and analysis. PYH contributed to initial optogenetic and immunostaining experiments. $\mathrm{FCO}, \mathrm{CH}$, and MCA designed experiments. AN and BNO assisted in some of the experiment design. All authors wrote the manuscript. MCA supervised the project.

\section{Acknowledgments}

We thank Manon Omnes (INSERM U1128) for technical assistance and genotyping, the SCM Imaging Platform of the Saints-Pères Biomedical Sciences site of Paris Descartes University for confocal microscopy, and the Imaging Platform of the ICM (ICM Quant) for EM experiments. We also thank D. Ortolani (INSERM U1266) for her assistance in the initial g-ratio quantification. This work was supported by grants from Fondation pour la Recherche Médicale (FRM, Equipe FRM DEQ20150331681), Fondation pour l'aide à la recherche sur la sclérose en plaques (ARSEP) to MCA, Agence Nationale pour la Recherche (ANR-14CE13-0023-02) to MCA and BNO, and a subaward agreement from the University of Connecticut (UConn) with funds provided by grant RG-1612-26501 from the National Multiple Sclerosis Society (NMSS) to MCA and AN. This article's contents are solely the responsibility of the authors and do not necessarily represent the official views of UConn or the NMSS. FCO was the recipient of an FRM postdoctoral fellowship and is supported by the Chilean National Fund for Scientific and Technological Development (FONDECYT, Iniciacion 11160616). FCO, CH, and MG were recipients of ARSEP postdoctoral fellowships. MG was supported by postdoctoral fellowships: the "Investissements d'avenir" ANR-10-IAIHU-06 (IHU-A-ICM).

Address correspondence to: María Cecilia Angulo, Institute of Psychiatry and Neuroscience of Paris, INSERM U1266; Université Paris Descartes, Sorbonne Paris Cité; 102, rue de la Santé, 75014 Paris, France. Phone: 33.1.40789243; Email: maria-cecilia.angulo@parisdescartes.fr. 
1. Franklin RJM, Ffrench-Constant C. Regenerating CNS myelin - from mechanisms to experimental medicines. Nat Rev Neurosci. 2017;18(12):753-769.

2. Chang A, Tourtellotte WW, Rudick R, Trapp BD. Premyelinating oligodendrocytes in chronic lesions of multiple sclerosis. $N$ Engl J Med. 2002;346(3):165-173.

3. Miller RH, Mi S. Dissecting demyelination. Nat Neurosci. 2007;10(11):1351-1354.

4. Barres BA, Raff MC. Proliferation of oligodendrocyte precursor cells depends on electrical activity in axons. Nature. 1993;361(6409):258-260.

5. Demerens C, et al. Induction of myelination in the central nervous system by electrical activity. Proc Natl Acad Sci U S A. 1996;93(18):9887-9892.

6. Gibson EM, et al. Neuronal activity promotes oligodendrogenesis and adaptive myelination in the mammalian brain. Science. 2014;344(6183):1252304.

7. Li Q, Brus-Ramer M, Martin JH, McDonald JW. Electrical stimulation of the medullary pyramid promotes proliferation and differentiation of oligodendrocyte progenitor cells in the corticospinal tract of the adult rat. Neurosci Lett. 2010;479(2):128-133

8. Powers AS. Adult neurogenesis in mammals and nonmammals. Commentary on Kempermann G (2012): new neurons for 'survival of the fittest'. Nat Rev Neurosci 13:727-736. Brain Behav Evol. 2013;81(4):206-208.

9. Mitew S, et al. Pharmacogenetic stimulation of neuronal activity increases myelination in an axon-specific manner. Nat Commun. 2018;9(1):306.

10. Wake H, Ortiz FC, Woo DH, Lee PR, Angulo MC, Fields RD. Nonsynaptic junctions on myelinating glia promote preferential myelination of electrically active axons. Nat Commun. 2015;6:7844.

11. Gautier HO, et al. Neuronal activity regulates remyelination via glutamate signalling to oligodendrocyte progenitors. Nat Commun. 2015;6:8518

12. Stevens B, Tanner S, Fields RD. Control of myelination by specific patterns of neural impulses. J Neurosci. 1998;18(22):9303-9311.

13. Stevens B, Porta S, Haak LL, Gallo V, Fields RD. Adenosine: a neuron-glial transmitter promoting myelination in the CNS in response to action potentials. Neuron. 2002;36(5):855-868.

14. Nagy B, Hovhannisyan A, Barzan R, Chen TJ, Kukley M. Different patterns of neuronal activity trigger distinct responses of oligodendrocyte precursor cells in the corpus callosum. PLoS Biol. 2017;15(8):e2001993.

15. Arenkiel BR, et al. In vivo light-induced activation of neural circuitry in transgenic mice expressing channelrhodopsin-2. Neuron. 2007;54(2):205-218.

16. Palmer LM, Stuart GJ. Site of action potential initiation in layer 5 pyramidal neurons. J Neurosci. 2006;26(6):1854-1863.

17. Angulo MC, Staiger JF, Rossier J, Audinat E. Distinct local circuits between neocortical pyramidal cells and fast-spiking interneurons in young adult rats. $J$ Neurophysiol. 2003;89(2):943-953.

18. Gensert JM, Goldman JE. Endogenous progenitors remyelinate demyelinated axons in the adult CNS. Neuron. 1997;19(1):197-203.

19. Sahel A, Ortiz FC, Kerninon C, Maldonado PP, Angulo MC, Nait-Oumesmar B. Alteration of synaptic connectivity of oligodendrocyte precursor cells following demyelination. Front Cell Neurosci. 2015;9:77.

20. Miron VE, et al. M2 microglia and macrophages drive oligodendrocyte differentiation during CNS remyelination. Nat Neurosci. 2013;16(9):1211-1218

21. Remaud S, et al. Transient hypothyroidism favors oligodendrocyte generation providing functional remyelination in the adult mouse brain. Elife. 2017;6:e29996.

22. Hill RA, Patel KD, Goncalves CM, Grutzendler J, Nishiyama A. Modulation of oligodendrocyte generation during a critical temporal window after NG2 cell division. Nat Neurosci. 2014;17(11):1518-1527.

23. Zonouzi M, et al. GABAergic regulation of cerebellar NG2 cell development is altered in perinatal white matter injury. Nat Neurosci. 2015;18(5):674-682.

24. Hughes EG, Orthmann-Murphy JL, Langseth AJ, Bergles DE. Myelin remodeling through experience-dependent oligodendrogenesis in the adult somatosensory cortex. Nat Neurosci. 2018;21(5):696-706.

25. Baraban M, Mensch S, Lyons DA. Adaptive myelination from fish to man. Brain Res. 2016;1641(pt A):149-161.

26. Wake H, Lee PR, Fields RD. Control of local protein synthesis and initial events in myelination by action potentials. Science. 2011;333(6049):1647-1651.

27. Hines JH, Ravanelli AM, Schwindt R, Scott EK, Appel B. Neuronal activity biases axon selection for myelination in vivo. Nat Neurosci. 2015;18(5):683-689.

28. Maldonado PP, Angulo MC. Multiple modes of communication between neurons and oligodendrocyte precursor cells. Neuroscientist. 2015;21(3):266-276.

29. Huang Y, Dreyfus CF. The role of growth factors as a therapeutic approach to demyelinating disease. Exp Neurol. 2016;283(pt B):531-540

30. Lundgaard I, et al. Neuregulin and BDNF induce a switch to NMDA receptor-dependent myelination by oligodendrocytes. PLoS Biol. 2013;11(12):e1001743.

31. Voronova A, et al. Migrating interneurons secrete fractalkine to promote oligodendrocyte formation in the developing mammalian brain. Neuron. 2017;94(3):500-516.e9.

32. Ishibashi T, et al. Astrocytes promote myelination in response to electrical impulses. Neuron. 2006;49(6):823-832.

33. Domingues HS, Portugal CC, Socodato R, Relvas JB. Oligodendrocyte, astrocyte, and microglia crosstalk in myelin development, damage, and repair. Front Cell Dev Biol. 2016;4:71.

34. Erlanger J, Gasser H. The action potential in fibers of slow conduction in spinal roots and somatic nerves. Am J Physiol. 1930;92(1):43-82

35. Gasser H, Erlanger J. The role played by the sizes of the constituent fibers of a nerve trunk in determining the form of its action potential wave. Am J Physiol. 1927;80(3):522-547.

36. Dimou L, Gallo V. NG2-glia and their functions in the central nervous system. Glia. 2015;63(8):1429-1451.

37. Green AJ, et al. Clemastine fumarate as a remyelinating therapy for multiple sclerosis (ReBUILD): a randomised, controlled, double-blind, crossover trial. Lancet. 2017;390(10111):2481-2489.

38. Bechler ME, Swire M, Ffrench-Constant C. Intrinsic and adaptive myelination-A sequential mechanism for smart wiring in the 
brain. Dev Neurobiol. 2018;78(2):68-79.

39. Abboud H, Hill E, Siddiqui J, Serra A, Walter B. Neuromodulation in multiple sclerosis. Mult Scler. 2017;23(13):1663-1676.

40. Berk C, Carr J, Sinden M, Martzke J, Honey CR. Thalamic deep brain stimulation for the treatment of tremor due to multiple sclerosis: a prospective study of tremor and quality of life. J Neurosurg. 2002;97(4):815-820.

41. Oliveria SF, et al. Safety and efficacy of dual-lead thalamic deep brain stimulation for patients with treatment-refractory multiple sclerosis tremor: a single-centre, randomised, single-blind, pilot trial. Lancet Neurol. 2017;16(9):691-700.

42. Cordella R, Franzini A, La Mantia L, Marras C, Erbetta A, Broggi G. Hypothalamic stimulation for trigeminal neuralgia in multiple sclerosis patients: efficacy on the paroxysmal ophthalmic pain. Mult Scler. 2009;15(11):1322-1328.

43. Centonze D, et al. Repetitive transcranial magnetic stimulation of the motor cortex ameliorates spasticity in multiple sclerosis Neurology. 2007;68(13):1045-1050.

44. Centonze D, et al. Effects of motor cortex rTMS on lower urinary tract dysfunction in multiple sclerosis. Mult Scler. 2007;13(2):269-271.

45. Koch G, Rossi S, Prosperetti C, Codecà C, Monteleone F, Petrosini L, Bernardi G, Centonze D. Improvement of hand dexterity following motor cortex rTMS in multiple sclerosis patients with cerebellar impairment. Mult Scler. 2008;14(7): 995-998.

46. Palm U, Ayache SS, Padberg F, Lefaucheur JP. Non-invasive brain stimulation therapy in multiple sclerosis: a review of tDCS, rTMS and ECT results. Brain Stimul. 2014;7(6):849-854.

47. Balia M, Benamer N, Angulo MC. A specific GABAergic synapse onto oligodendrocyte precursors does not regulate cortical oligodendrogenesis. Glia. 2017;65(11):1821-1832.

48. R Core Team. R: A language and environment for statistical computing. R Foundation for Statistical Computing. Vienna, Austria. http://www.R-project.org/. Accessed April 2, 2019. 